\title{
Clinical, Endocrinological and Immunological Characteristics of Japanese Patients With Autoimmune Polyglandular Syndrome Type $3 a$
}

\author{
Sumie Moriyama ${ }^{\text {a }}$, Reo Yoshikawa ${ }^{\text {a }}$, Hisayuki Katsuyama ${ }^{a}$, Hidetaka Hamasaki ${ }^{a}$, Hiroki Adachi ${ }^{\text {a }}$, \\ Hidekatsu Yanai ${ }^{a, b}$
}

\begin{abstract}
Background: Autoimmune polyglandular syndrome (APS) is an autoimmune disease that involves multiple organ failure. In APS3, autoimmune thyroid disease occurs with other autoimmune diseases, but not with Addison disease. APS3a is defined as APS3 including autoimmune diabetes. The information about clinical backgrounds of APS3a is very limited. We studied to understand clinical, endocrinological and immunological characteristics of Japanese patients with APS3a.
\end{abstract}

Methods: We reviewed our previously published case reports about APS3a, and picked up patients with type 1 diabetes and autoimmune diabetes, who showed the positivity for anti-glutamic acid decarboxylase antibody (anti-GAD ab) or anti-islet antigen 2 antibody (anti-IA2 $\mathrm{ab)}$ or anti-thyroglobulin antibody (anti-TG ab) or anti-thyroid peroxidase antibody (anti-TPO ab) or anti-thyroid stimulating hormone receptor antibody (anti-TR ab) between January 2010 and January 2016. We collected data including age, sex, anti-GAD ab, anti-IA2 ab, $\mathrm{HbAlc}$, serum fasting C-peptide immunoreactivity (CPR), urinary CPR, treatment for diabetes, anti-TPO ab, anti-TG ab, anti-TR ab, thyroid stimulating hormone (TSH), free triiodothyronine (fT3), free thyroxine (fT4), findings of thyroid ultrasonography and treatment for thyroid diseases.

Results: Present study revealed a remarkable female predominance in APS3a. Among patients with autoimmune diabetes, slowly progressive insulin-dependent diabetes mellitus (SPIDDM) was the most common type of diabetes, and almost $80 \%$ of patients with APS3a showed the positivity for anti-GAD ab. Among patients with autoimmune thyroid diseases, almost $80 \%$ of patients had possible Hashimoto thyroiditis. Almost $70 \%$ of patients with APS3a showed the positivity for anti-TPO ab. Almost $20 \%$ of

Manuscript accepted for publication March 23, 2016

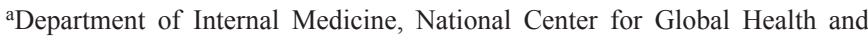
Medicine Kohnodai Hospital, Chiba, Japan

${ }^{\mathrm{b}}$ Corresponding Author: Hidekatsu Yanai, Department of Internal Medicine, National Center for Global Health and Medicine Kohnodai Hospital, 1-7-1 Kohnodai, Ichikawa, Chiba 272-0034, Japan.

Email: dyanai@hospk.ncgm.go.jp

doi: http://dx.doi.org/10.14740/jem348e
APS3a patients had possible Grave's disease, and $83 \%$ of patients with possible Grave's disease showed overt Grave's disease. In patients with possible Grave's disease, the positive rate of anti-TR ab was $100 \%$.

Conclusion: We revealed clinical, endocrinological and immunological characteristics of patients with APS3a in Japan.

Keywords: Autoimmune polyglandular syndrome 3a; Insulin; Thyroid diseases; Type 1 diabetes

\section{Introduction}

Autoimmune polyglandular syndrome (APS) was defined as an autoimmune disease that involves multiple organ failure $[1,2]$. APS was classified into four types, and APS types 1 , 2 and 4 include autoimmune adrenalitis, Addison disease, as major components of disease [3-5]. In APS3, autoimmune thyroiditis occurs with other autoimmune diseases, but not with Addison disease [3,4]. APS3 is further classified into $3 a, 3 b$ and $3 c$. APS3a, APS3b and APS3c are defined as APS3 including autoimmune diabetes, pernicious anemia, vitiligo and alopecia as major components of disease, respectively $[3,4]$. Autoimmune thyroid disease is the most common ( $>$ $90 \%$ ) organ-specific autoimmune disease that develops as a complication among patients with type 1 diabetes in Japan [5, 6]. Therefore, APS3a may be the most common type of APS in Japan.

Although case reports about APS3a including ours were found by the search using Pubmed [4, 7-9], the information about clinical backgrounds of APS3a is very limited. Only one article reported clinical and genetic characteristics of adult APS3, and we found one more article which reported the incidence of APS 3 in children with type 1 diabetes $[10,11]$.

To understand clinical, endocrinological and immunological characteristics of Japanese patients with APS3a, we reviewed and summarized our previous four case reports about APS3a at first. Furthermore, we showed clinical backgrounds of 26 Japanese patients with APS3a that we accumulated, and we analyzed data of these patients to find characteristic features for APS3a in Japan. 
Table 1. Clinical, Endocrinological and Immunological Characteristics of Our Previously Reported Japanese Patients With Autoimmune Polyglandular Type 3a

\begin{tabular}{|c|c|c|c|c|c|c|c|}
\hline $\begin{array}{l}\text { Cases } \\
\text { [Reference] }\end{array}$ & $\begin{array}{l}\text { Age } \\
\text { (years)/sex }\end{array}$ & $\begin{array}{l}\text { Type of } \\
\text { diabetes }\end{array}$ & $\begin{array}{l}\text { Anti-GAD } \\
\text { antibody }\end{array}$ & $\begin{array}{l}\text { Urinary C- } \\
\text { peptide }(\mu \mathrm{g} / \text { day })\end{array}$ & $\begin{array}{l}\text { Positivity for anti-TPO } \\
\text { or anti-TG antibody }\end{array}$ & $\begin{array}{l}\text { Thyroid } \\
\text { function }\end{array}$ & Other complication \\
\hline Case 1 [4] & $53 / \mathrm{F}$ & SPIDDM & 10,000 & 70 & $(+)$ & Euthyroid & $\begin{array}{l}\text { Symptomatic Sjogren's } \\
\text { syndrome with anti- } \\
\text { SSA/Ro ab }(+) \text { and } \\
\text { anti-SSB/La ab }(+) \text {; } \\
\text { asymptomatic RA with } \\
\text { RF }(+)\end{array}$ \\
\hline Case 3 [8] & $68 / \mathrm{F}$ & SPIDDM & 34 & 52.9 & $(+)$ & Euthyroid & None \\
\hline Case 4 [9] & $84 / \mathrm{F}$ & SPIDDM & 65.2 & $<0.3$ & $(-)$ & $\begin{array}{l}\text { Treated by } \\
\text { levothyroxine } \\
(50 \mu \mathrm{g} / \text { day })\end{array}$ & $\begin{array}{l}\text { Sarcoidosis, candidiasis, } \\
\text { and liver cirrhosis due } \\
\text { to autoimmune hepatitis }\end{array}$ \\
\hline
\end{tabular}

F: female; GAD: glutamic acid decarboxylase; MRHE: mineralocorticoid-responsive hyponatremia with the elderly; RA: rheumatoid arthritis; ref: reference; RF: rheumatoid factor; SPIDDM: slowly progressive insulin-dependent diabetes mellitus; TG: thyroglobulin; TPO: thyroid peroxidase. Normal ranges of anti-GAD antibody and urinary C-peptide are $<1.5 \mathrm{U} / \mathrm{mL}$ and $29.2-167.0 \mu \mathrm{g} /$ day, respectively.

\section{Materials and Methods}

\section{Review and summarization of our previously published case reports about APS3a}

We reviewed four our previously published case reports about APS3a, and showed data about age, sex, type of diabetes, anti-glutamic acid decarboxylase antibody (anti-GAD ab), the positivity for anti-thyroglobulin antibody (anti-TG ab) or antithyroid peroxidase antibody (anti-TPO ab), thyroid function and other complication in 4 patients with APS3a.

\section{Subjects}

This study was approved by the Institutional Ethics Committee in National Center for Global Health and Medicine (NCGMG-001947-00), and was also performed in accordance with the Declaration of Helsinki.

We picked up patients with type 1 diabetes and autoimmune diabetes, who showed positivity for anti-GAD ab or anti-islet antigen 2 antibody (anti-IA2 ab) or anti-TG ab or antiTPO ab or anti-thyroid stimulating hormone receptor antibody (anti-TR ab) between January 2010 and January 2016.

\section{Data collection}

We collected data including age, sex, anti-GAD ab, anti-IA2 $\mathrm{ab}, \mathrm{HbA} 1 \mathrm{c}$, serum fasting C-peptide immunoreactivity (CPR), urinary CPR, treatment for diabetes, anti-TPO ab, anti-TG ab, anti-TR ab, thyroid stimulating hormone (TSH), free triiodothyronine (fT3), free thyroxine (fT4), findings of thyroid ultrasonography and treatment for thyroid diseases.

\section{Definition of autoimmune thyroid diseases}

Grave's disease was defined as a history of primary hyperthyroidism with positive anti-TR ab, and Hashimoto thyroiditis was defined as having diffuse goiter and/or primary hypothyroidism with positive anti-TPO ab or positive anti-TG ab [12]. However, we have previously found a significant discrepancy between endocrinological phenotype and immunological phenotype in APS3a [4]. In present study, a history of primary hyperthyroidism with positive anti-TR ab was defined as overt Grave's disease, and euthyroidism with positive anti-TR ab was defined as immunological Grave's disease. We defined overt Grave's disease and immunological Grave's disease as possible Grave's disease. Having diffuse goiter and/or primary hypothyroidism with positive anti-TPO ab or positive anti-TG ab was defined as overt Hashimoto thyroiditis, and the absence of diffuse goiter and/or primary hypothyroidism with positive anti-TPO ab or positive anti-TG ab was defined as immunological Hashimoto thyroiditis. Subclinical hypothyroidism was diagnosed when peripheral thyroid hormone levels were within normal reference laboratory range but serum TSH levels were mildly elevated [13]. We defined overt Hashimoto thyroiditis and immunological Hashimoto thyroiditis and subclinical hypothyroidism with positive antiTPO $\mathrm{ab}$ or positive anti-TG ab as possible Hashimoto thyroiditis.

\section{Results}

Table 1 shows clinical, endocrinological and immunological characteristics of four our previously reported Japanese patients with ASP3a [4, 7-9]. All patients were female, and age ranged from 53 to 84 years old. Type of diabetes in all patients was slowly progressive insulin-dependent diabetes mellitus 


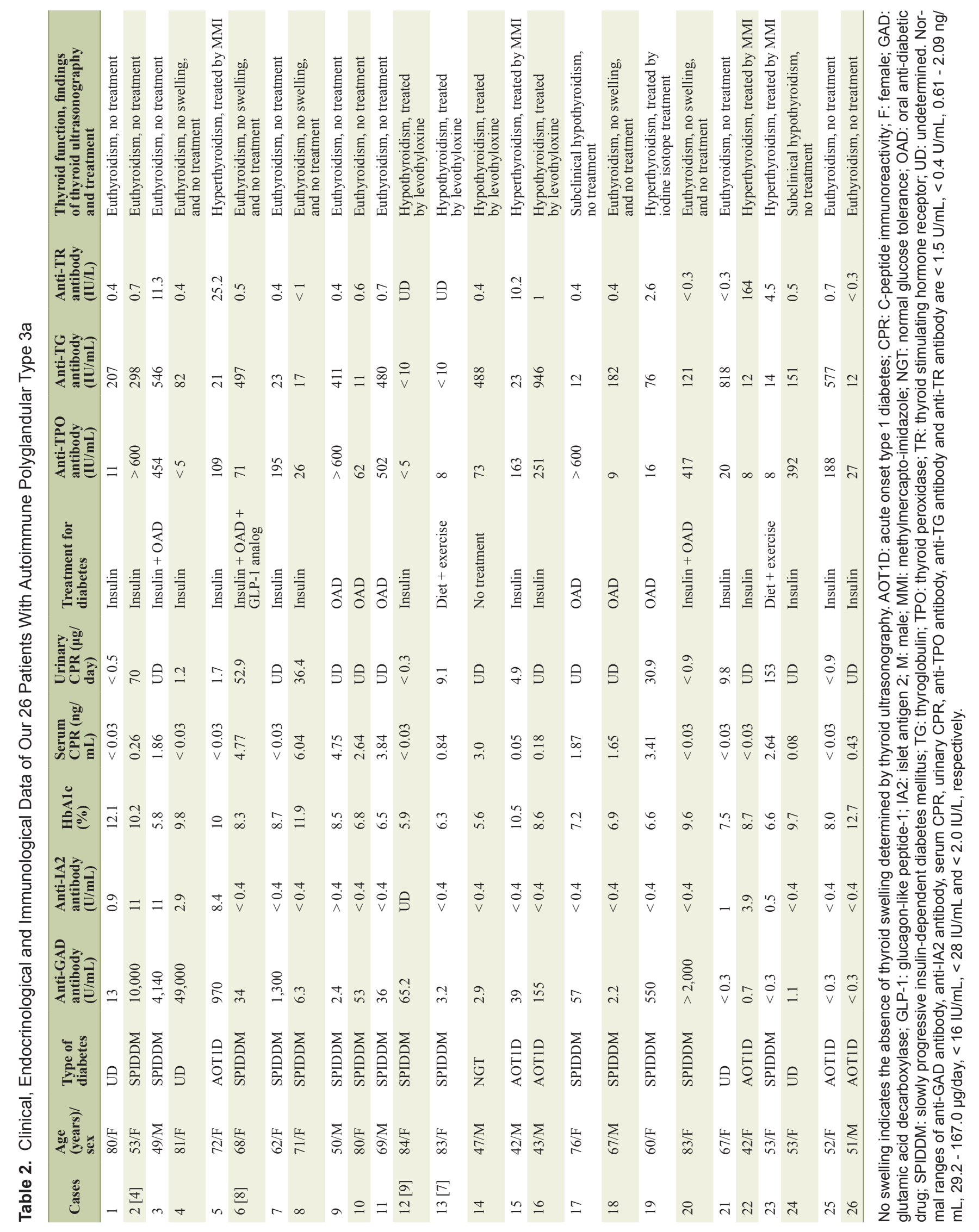


Table 3. Sex, Type of Diabetes or Glucose Intolerance, the Positivity for Diabetes-Associated Autoantibodies, Treatment for Diabetes, Kinds and Thyroid Function of Autoimmune Thyroid Diseases and the Positivity for Thyroid-Associated Autoantibodies in Patients With Autoimmune Polyglandular Type 3a in Japan

\begin{tabular}{|c|c|}
\hline Sex (male/female) & $8 / 18$ \\
\hline \multicolumn{2}{|l|}{ Autoimmune diabetes } \\
\hline SPIDDM & $15 / 26(57.7 \%)$ \\
\hline Acute onset type 1 diabetes & $6 / 26(23.1 \%)$ \\
\hline Normal glucose tolerance & $1 / 26(3.8 \%)$ \\
\hline Anti-GAD antibody $(+)$ & $20 / 26(76.9 \%)$ \\
\hline Anti-IA2 antibody $(+)$ & $8 / 26(30.8 \%)$ \\
\hline \multicolumn{2}{|l|}{ Treatment for diabetes } \\
\hline Insulin & $14 / 26(53.8 \%)$ \\
\hline Insulin + OAD & $2 / 26(7.7 \%)$ \\
\hline Insulin + OAD + GLP-1 analog & $1 / 26(3.8 \%)$ \\
\hline $\mathrm{OAD}$ & $6 / 26(23.1 \%)$ \\
\hline Diet + exercise & $2 / 26(7.7 \%)$ \\
\hline No treatment & $1 / 26(3.8 \%)$ \\
\hline \multicolumn{2}{|l|}{ Autoimmune thyroid diseases } \\
\hline Possible Hashimoto thyroiditis & $21 / 26(80.8 \%)$ \\
\hline Overt Hashimoto thyroiditis & $4 / 26(15.4 \%)$ \\
\hline Subclinical hypothyroidism due to Hashimoto thyroiditis & $2 / 26(7.7 \%)$ \\
\hline Immunological Hashimoto thyroiditis & $15 / 26(57.7 \%)$ \\
\hline Anti-TPO antibody $(+)$ & $19 / 26(73.1 \%)$ \\
\hline Anti-TG antibody $(+)$ & $15 / 26(57.7 \%)$ \\
\hline Possible Graves's disease & $6 / 26(23.1 \%)$ \\
\hline Overt Grave's disease & $5 / 26(19.2 \%)$ \\
\hline Immunological Grave's disease & $1 / 26(3.8 \%)$ \\
\hline Anti-TR antibody $(+)$ & $6 / 26(23.1 \%)$ \\
\hline
\end{tabular}

GAD: glutamic acid decarboxylase; GLP-1: glucagon-like peptide-1; IA2: islet antigen 2; OAD: oral anti-diabetic drug; SPIDDM: slowly progressive insulin-dependent diabetes mellitus; TG: thyroglobulin; TPO: thyroid peroxidase; TR: thyroid stimulating hormone receptor.

(SPIDDM). Anti-GAD ab levels ranged widely from 3.2 to $10,000 \mathrm{U} / \mathrm{mL}$. Beta-cell function was preserved in two patients and was severely disturbed in two patients. Autoimmune thyroid disease in all patients was Hashimoto thyroiditis, and two patients showed euthyroidism and two patients were treated by levothyroxine. We found Sjogren's syndrome, elevated rheumatoid factor, mineralocorticoid-responsive hyponatremia with the elderly (MRHE), sarcoidosis, candidiasis, and liver cirrhosis due to autoimmune hepatitis as complications of APS3a.

We found 26 patients with APS3a. Clinical, endocrinological and immunological data of our patients with APS3a are shown in Table 2. We summarized 26 patients' data including sex, type of diabetes or glucose intolerance, the positivity for diabetes-associated autoantibodies, treatment for diabetes, kinds and thyroid function of autoimmune thyroid diseases, and the positivity for thyroid-associated autoantibodies in patients with APS3a in Japan (Table 3).
Mean \pm SD and range of age, HbA1c, and levels of antiGAD ab, anti-IA2 ab, serum fasting CPR, urinary CPR, antiTPO ab, anti-TG ab and anti-TR ab in patients with APS3a in Japan are shown in Table 4.

\section{Discussion}

We previously found and reported a significant discrepancy between endocrinological and clinical phenotype and immunological phenotype in a female patient with APS3a [4]. In spite of her high level of anti-TPO ab and anti-TG ab, she did not show diffuse goiter and primary hypothyroidism. Further, in spite of remarkable high level of anti-GAD ab (10,000 U/ $\mathrm{mL}$ ), her beta-cell function was preserved when she was diagnosed as having APS3a. After several months, she showed an insulin-dependent state. We also experienced a female patient showing the absence of diffuse goiter and primary 
Table 4. Mean $\pm \mathrm{SD}$ and Range of Age, HbA1c, and Levels of Anti-GAD Antibody, Anti-IA2 Antibody, Serum Fasting CPR, Urinary CPR, Anti-TPO Antibody, Anti-TG Antibody and Anti-TR Antibody in Patients With Autoimmune Polyglandular Type 3a in Japan

\begin{tabular}{lll}
\hline & Mean \pm SD & Range \\
\hline Age & $63.0 \pm 14.2$ & $42-86$ \\
HbA1c & $8.4 \pm 2.0$ & $5.6-12.7$ \\
Anti-GAD antibody $(\mathrm{U} / \mathrm{mL})$ & $2,632.0 \pm 9,685.0$ & $<0.3-49,000$ \\
Anti-IA2 antibody $(\mathrm{U} / \mathrm{mL})$ & $1.86 \pm 3.26$ & $<0.4-11.0$ \\
Serum CPR $(\mathrm{ng} / \mathrm{mL})$ & $1.48 \pm 1.84$ & $<0.03-3.84$ \\
Urinary CPR $(\mu \mathrm{g} / \mathrm{day})$ & $29.9 \pm 55.2$ & $<0.5-153$ \\
Anti-TPO antibody $(\mathrm{IU} / \mathrm{mL})$ & $185.4 \pm 215.7$ & $<5.0->600$ \\
Anti-TG antibody $(\mathrm{IU} / \mathrm{mL})$ & $232.5 \pm 275.1$ & $<10.0-946$ \\
Anti-TR antibody $(\mathrm{IU} / \mathrm{L})$ & $9.47 \pm 33.4$ & $<0.3-25.2$ \\
TSH & $3.54 \pm 6.09$ & $<0.03-29.91$ \\
fT3 & $3.65 \pm 2.03$ & $2.09-5.92$ \\
fT4 & $1.15 \pm 0.75$ & $0.67-1.74$ \\
\hline
\end{tabular}

When we determined mean \pm SD of each variable including variables under the lower limit and above the upper limit, we used variables of upper and lower limit. CPR: C-peptide immunoreactivity; GAD: glutamic acid decarboxylase; fT4: free thyroxine; fT3: free triiodothyronine; IA2: islet antigen 2; TG: thyroglobulin; TPO: thyroid peroxidase; TR: thyroid stimulating hormone receptor; $\mathrm{TSH}$ : thyroid stimulating hormone. Normal ranges of anti-GAD antibody, anti-IA2 antibody, serum CPR, urinary CPR, anti-TPO antibody, anti-TG antibody, anti-TR antibody, TSH, fT3 and fT4 are $<1.5 \mathrm{U} / \mathrm{mL},<0.4 \mathrm{U} / \mathrm{mL}, 0.61-2.09 \mathrm{ng} / \mathrm{mL}, 29.2$ - $167.0 \mu \mathrm{g} /$ day, $<16 \mathrm{IU} / \mathrm{mL},<28 \mathrm{IU} / \mathrm{mL},<2.0 \mathrm{IU} / \mathrm{L}, 0.54-4.26 \mu \mathrm{lU} / \mathrm{mL}$, $2.39-4.06 \mathrm{pg} / \mathrm{mL}$ and $0.71-1.52 \mathrm{ng} / \mathrm{dL}$, respectively.

hypothyroidism with high level of anti-TPO ab and anti-TG $\mathrm{ab}$ [8]. Therefore, we defined the absence of diffuse goiter and/or primary hypothyroidism with positive anti-TPO ab or positive anti-TG ab as immunological Hashimoto thyroiditis. We also found an insulin-dependent female patient (urinary $\mathrm{CPR}<0.3 \mu \mathrm{g} /$ day) with positive anti-GAD antibody [9]. She has hypothyroidism treated by levothyroxine; however, both anti-TPO ab and anti-TG ab were negative. To grasp APS3a in detail, we picked up patients with type 1 diabetes and autoimmune diabetes showing the positivity for anti-GAD ab or anti-IA2 ab or anti-TG ab or anti-TPO ab or anti-TR ab as patients with APS3a. Both anti-GAD ab and anti-IA2 ab were negative in cases 24, 25 and 26 in Table 2; however, they were insulin-dependent and showed the positivity for antiTPO ab and anti-TG ab, and they were included in patients with APS3a.

Our previous reports [4, 7-9] and present study revealed a remarkable female predominance in APS3a, which agreed with previous reports $[10,11]$. Among patients with autoimmune diabetes, SPIDDM was the most common type of diabetes, and the positive rate of anti-GAD ab was higher than that of anti-IA2 ab. Almost $80 \%$ of patients with APS3a showed the positivity for anti-GAD ab. Almost $65 \%$ patients were treated by using insulin, and approximately $23 \%$ and $8 \%$ of patients were treated by oral anti-diabetic drugs and diet/ exercise, respectively. Serum and urinary CPR ranged widely from undetectable level to hyperinsulinemic level, suggesting that patients with APS3a include insulin-dependent and hyperinsulinemic patients. These may be due to the predominance of SPIDDM as phenotype of diabetes in APS3a.

Among patients with autoimmune thyroid diseases, almost $80 \%$ of patients had possible Hashimoto thyroiditis. In patients with possible Hashimoto thyroiditis, immunological Hashimoto thyroiditis was the most common phenotype. The positive rate of anti-TPO ab was higher than that of anti-TG ab. Almost $70 \%$ of patients with APS3a showed the positivity for anti-TPO ab. Overt Hashimoto thyroiditis defined by having diffuse goiter and/or primary hypothyroidism with positive anti-TPO ab or positive anti-TG ab was observed in only $15 \%$ of APS3a patients. Hashimoto thyroiditis may also progress slowly, which was supported by the predominance of SPIDDM as phenotype of diabetes in APS3a.

Almost $20 \%$ of patients had possible Grave's disease, and $83 \%$ of patients with possible Grave's disease showed overt Grave's disease. In patients with possible Grave's disease, the positive rate of anti-TR antibody was $100 \%$.

In this study, $92.3 \%$ of APS3a patients showed the positivity for anti-GAD ab or anti-TPO ab, which suggests that the measurement of anti-GAD ab or anti-TPO ab is the useful tool to detect APS3a.

We have to mention the limitation of our study. Although APS3a is very rare, the number of subjects studied was small. To elucidate the pathogenesis of APS3a, further studies, preferably with larger numbers of subjects, will be needed. Further, we should follow up the untreated patients with positive diabetes and thyroid-associated autoantibodies.

\section{Conclusion}

We revealed clinical, endocrinological and immunological characteristics of patients with APS3a in Japan.

\section{Author Contributions}

S.M. and H.Y. designed the research. R.Y., H.K., H.H., H.A. and H.Y. collected data. S.M. and H.Y. analyzed data, and wrote the paper. All authors read and approved the final paper.

\section{Conflict of Interests}

The authors declare that they have no conflicts of interest concerning this article.

\section{References}

1. Neufeld M, Maclaren N, Blizzard R. Autoimmune polyglandular syndromes. Pediatr Ann. 1980;9(4):154-162.

2. Anderson MS. Update in endocrine autoimmunity. J Clin Endocrinol Metab. 2008;93(10):3663-3670. 
3. Betterle C, Dal Pra C, Mantero F, Zanchetta R. Autoimmune adrenal insufficiency and autoimmune polyendocrine syndromes: autoantibodies, autoantigens, and their applicability in diagnosis and disease prediction. Endocr Rev. 2002;23(3):327-364.

4. Hamasaki H, Yanai H, Hiraishi C, Moriyama S. A significant discrepancy between endocrinological and clinical phenotype and immunological phenotype in autoimmune polyglandular syndrome type 3 . J Med Cases. 2012;3(1):1-3.

5. Eisenbarth GS, Gottlieb PA. Autoimmune polyendocrine syndromes. N Engl J Med. 2004;350(20):2068-2079.

6. Gray RS, Herd R, Clarke BF. The clinical features of diabetes with coexisting autoimmune thyroid disease. Diabetologia. 1981;20(6):602-606.

7. Yanai H, Okamoto S, Kunimatsu J. Autoimmune polyglandular syndrome type 3 complicated by mineralocorticoid-responsive hyponatremia of the elderly. World J Diabetes. 2010;1(4):135-136.

8. Moriyama S, Yanai $H$. The addition of metformin and glucagon-like peptide-1 receptor agonist or dipeptidylpeptidase-4 inhibitor to insulin for latent autoimmune di- abetes in adults. Int J Diabetes Dev Ctries 2014; 34(1):56.

9. Yoshikawa R, Sako A, Kitagawa H, Hamasaki H, Okubo T, Hiraishi C, Suzuki K, Mishima S, Yanai H. The development of central pontine myelinolysis in a type 1 diabetic patient with anti-insulin antibody and similar clinical manifestations to autoimmune polyglandular syndrome. J Endocrinol Metab. 2012;2(4-5):181-186.

10. Horie I, Kawasaki E, Ando T, Kuwahara H, Abiru N, Usa $\mathrm{T}$, Yamasaki H, et al. Clinical and genetic characteristics of autoimmune polyglandular syndrome type 3 variant in the Japanese population. J Clin Endocrinol Metab. 2012;97(6):E1043-1050.

11. Ben-Skowronek I, Michalczyk A, Piekarski R, WysockaLukasik B, Banecka B. Type III Polyglandular Autoimmune Syndromes in children with type 1 diabetes mellitus. Ann Agric Environ Med. 2013;20(1):140-146.

12. Kasai K. [The use and problems of diagnostic guidelines for thyroid diseases]. Nihon Naika Gakkai Zasshi. 2010;99(4):692-699.

13. Fatourechi V. Subclinical hypothyroidism: an update for primary care physicians. Mayo Clin Proc. 2009;84(1):6571. 